\title{
Predictores socioemocionales y cognitivos: su papel en la comprensión del envejecimiento con éxito en el contexto costarricense*
}

Socio-Emotional and Cognitive Predictors: Its Roll on the Comprehension of the Successful Aging in Costarica's Context

\author{
Mauricio Blanco Molina \\ Universidad Nacional de Costa Rica, Costa Rica \\ Mónica Salazar Villanea \\ Universidad de Costa Rica, Costa Rica
}

a Autor de correspondencia. Correo electrónico: mauricioblanco.molina@gmail.com

Para citar este artículo: Blanco Molina, M., \& Salazar Villanea, M. (2017). Predictores socioemocionales y cognitivos: su papel en la comprensión del envejecimiento con éxito en el contexto costarricense. Universitas Psychologica, 16(1), 1-16. http://dx.doi.org /10.11144/Javeriana.upsy16-1.pscc

\section{RESUMEN}

Tomando en cuenta las teorías del envejecimiento con éxito, este estudio se orientó hacia la identificación y el análisis de las características que presentan personas adultas mayores con altos niveles de actividad y participación social residentes en Costa Rica. El estudio contó con 191 participantes con edades entre 55 y 87 años $(M=68.5, D E=6.9)$ que habitan en el área metropolitana y participan en programas dirigidos a personas mayores en cursos de computación, idiomas, actividad física, arte, entre otros. Mediante modelos lineales, se evidenció la importancia de las condiciones sociales, emocionales y cognitivas como las mejores variables predictoras del nivel de actividad en la muestra estudiada: a) variables emocionales como autoeficacia percibida, emociones positivas, satisfacción con la vida, control personal, adecuadas redes de apoyo; b) características cognitivas como funciones ejecutivas preservadas; c) percepción subjetiva de salud y d) características sociodemográficas como nivel educativo e ingresos. El estudio permite funcionar como línea base, a nivel teórico y metodológico, del envejecimiento con éxito en Costa Rica. Describe los estilos de vida activos en la vejez de los participantes costarricenses, caracterizados primordialmente por la participación en relaciones sociales y ocio, más que por actividades de tipo cognitivas y físicas o vinculadas al logro personal.

Palabras clave

persona adulta mayor; variables socioemocionales; variables cognitivas; envejecimiento con éxito.

\begin{abstract}
The present study was aimed at identifying and analyzing the characteristics of older people with high levels of activity and social participation living in Costa Rica, from perspectives like the Successful Aging approach. It also addresses the classical criteria of successful aging theories and their scope in Costa Rican culture. 191 participants between the ages of 55 and $87(M=68.5, S D=6.9)$ who live in the metropolitan area and participate in programs of the Costa Rican Gerontological Association and the Program for the older people at the University of Costa Rica (in computer courses, languages courses, physical activity, art, among others). Linear models analysis has shown that social, emotional and cognitive factors are the best predictors of the level of activity in
\end{abstract}


the analyzed sample. The variables combined to better predict social participation in leisure activities, physical activities, cognitive activities, instrumental functionality and productivity in old age are: a.) Variables of emotional stability as self-efficacy, positive emotions, life satisfaction, personal control, adequate support networks b.) executive functions, c.) perceived subjective health and d.) sociodemographic characteristics such as level of education and income. The study can serve as a baseline for theoretical and methodical descriptions of successful aging in Costa Rica, characterized by a strong orientation to give more importance to social participation and leisure activities rather than cognitive and physical activity or personal achievement.

Keywords

older people; socio-emotional variables; cognitive variables; successful aging.

\section{Introducción}

A partir de una perspectiva psicosocial, la construcción teórico-conceptual del proceso de envejecimiento exitoso ha liderado desde hace aproximadamente 30 años el conocimiento científico psicogerontológico, con efectos en la investigación básica y aplicada a nivel mundial (Bowling \& Dieppe, 2005; Bowling \& Ilieffe, 2006, 2011; Bülow \& Söderqvist, 2014; Cho, Martin, \& Poon, 2015; Freund, Nikitin, \& Ritter, 2009; Johnson, Sarkisian, \& Williamson, 2015; Jopp et al., 2015; Kahana, Kahana, \& Lee, 2014; Ouwehand, de Ridder, \& Bensing, 2007; Steverink, Lindenberg, \& Ormel, 1998). El envejecimiento con éxito se ha estudiado como un concepto multidimensional que abarca, trasciende y supera la buena salud (Organización Mundial de la Salud [OMS], 2002), y que está compuesto por un amplio conjunto de factores biopsicosociales, reconociendo que existe gran variabilidad interindividual en las formas de envejecer (Bowling \& Ilieffe, 2011).

El presente trabajo investigó los determinantes y/o predictores del envejecimiento con éxito en una muestra de personas adultas mayores con alto nivel de participación residentes sociales en Costa Rica. Se priorizó, como en investigaciones previas, el análisis en la indagación de las variables socioemocionales (Eizenman, Nesselroade, Featherman, \& Rowe, 1997; Fernández-Ballesteros, Diez-Nicolás, Caprara,
Barbaranelli, \& Bandura, 2002; Freund \& Baltes, 1998; Jopp \& Smith, 2006; Lang \& Carstensen, 1994; Mendes de León, 2005; Seeman, McAvay, Merrill, Albert, \& Rodin, 1996) y cognitivas que predicen este fenómeno durante la vejez (Dockree, Brennan, O'Sullivan, Robertson, \& O'Connell, 2015; Hogan, Mata, \& Carstensen, 2013; Krueger, Wilson, Kamenetsky, Barnes, Bienias, \& Bennett, 2009; Vásquez, Rodríguez, Villarreal, \& Campos, 2014). Además, se buscó describir las particularidades que caracterizan a aquellas personas adultas mayores que mantienen un alto nivel de participación social y física dentro y fuera de sus hogares (Glei, Landau, Goldman, Chuang, Rodriguez, \& Weinstein, 2005; Hogan et al., 2013; Johnson et al., 2015; Potočnik \& Sonnentag, 2013), que ha sido definida en la publicación antecedente como criterio de clasificación del envejecimiento con éxito en distintos contextos y metodologías de análisis (Cho et al., 2015; Fernández-Ballesteros, Zamarrón, López, Molina, Díez, Montero, \& Schettini, 2010; Gureje, Oladeji, Abiona, \& Chatterji, 2014; Parslow, Lewis, \& Nay, 2011; Jopp, Wozniak, Damarin, De Feo, Jung, \& Jeswani, 2015; Kahana et al., 2014; Kahana, Kelley-Moore, \& Kahana, 2012). Se consideraron también variables asociadas a condiciones de salud (Haveman-Nies, de Groot, \& van Staveren, 2003; Tyrovolas, Haro, Mariolis, Piscopo, Valacchi, Tsakountakis, \& Metallinos, 2014) y características sociodemográficas (Gestorf et al., 2008) presentes en el contexto de la muestra estudiada (Blanco-Molina, 2010).

El aporte fundamental yace en la posibilidad de describir, con evidencia empírica, cómo se articulan las variables que componen los llamados recursos socioemocionales y cognitivos que permiten la adaptación y compensación en la vejez (Baltes \& Baltes, 1990; Jopp \& Smith, 2006; Schulz \& Heckhausen, 1996; Strawbridge, Cohen, Shema, \& Kaplan, 1996), buscando reflexionar sobre los criterios clásicos del envejecimiento con éxito (Bülow \& Söderqvist, 2014; Rowe \& Kahn, 1998), que son aspectos revisados con poca profundidad en estudios 
anteriores a nivel nacional (Rosero-Bixby, 2008; Rosero-Bixby \& Dow, 2005, 2009).

El estudio buscó responder las siguientes preguntas: ¿Qué características socioemocionales, cognitivas, de salud y sociodemográficas evidencia una persona adulta mayor con altos niveles de actividad residente en Costa Rica? y ¿Cuáles de las variables medidas predicen mejor los niveles de actividad presentados en la muestra estudiada?

El envejecimiento con éxito ha sido generalmente entendido como envejecer bien, tener una buena salud en la vejez, como un fenómeno deseable, individual y socialmente esperado. Su noción, sin embargo, varía según contexto, países y momento histórico, por lo que resulta muy difícil su identificación o medición: los indicadores subjetivos y objetivos de envejecimiento con éxito necesitan ser considerados dentro del contexto cultural donde viva el sujeto, con su contenido particular y sus demandas ecológicas (Baltes \& Baltes, 1990).

Desde los inicios de su aparición en la publicación científica, la psicología y la gerontología hicieron énfasis en superar las barreras de la geriatría dominante. Autores más clásicos, como Havighurst (1963) y Ryff (1989), argumentaban que el fenómeno se puede describir como la constante necesidad de disponer durante el ciclo vital de estrategias de flexibilidad y adaptación ante los distintos obstáculos, siendo relevante también contar con satisfacción con la vida. Para Kahana y Kahana (1996), este proceso debe promover la posibilidad de un continuo desarrollo. Por su parte, Baltes y Baltes (1990) lo definieron como punto de partida para aquellas personas que muestran muy poca o ninguna pérdida de funcionamiento comparativamente con el promedio de personas más jóvenes que ellos. Para Rowe y Kahn (1998), la vejez con éxito se expresaba como un enlace de la salud (biopsicosocial) con la habilidad funcional mantenida, adoptando estilos de vida saludables y manteniendo un funcionamiento cognitivo y físico con alto compromiso activo con la vida durante la vejez (sentimiento de utilidadde formar parte).
En otras palabras, el fenómeno fue descrito como el proceso de cada persona en el logro de un compromiso de autorregulación emocional, social, cognitiva y física que desencadena una adaptación en la adultez mayor. En ese sentido, también se argumenta la importancia de la presencia de patrones de control primario y secundario (Schulz \& Heckhausen, 1996) y la necesidad de establecer mecanismos de selectividad socioemocional (Baltes \& Carstensen, 1996). Sin buscar la exhaustividad en la descripción del fenómeno desde las descripciones psicológicas y gerontológicas más relevantes, a continuación, se describe brevemente el método utilizado en el estudio.

\section{Método}

\section{Diseño y participantes}

El presente trabajo siguió la línea de una investigación correlacional y transversal, bajo el marco de un modelo lineal. Para el análisis lineal, se definió el nivel de actividad como variable dependiente, medida a partir de un autorreporte de la frecuencia de ocupaciones de ocio, cognitivas, sociales, de tipo instrumental, productivas y físicas. Las variables independientes fueron los recursos o características personales de carácter biopsicosocial, identificados en los antecedentes científicos sobre el tema. Se seleccionó a los participantes mediante un muestreo no probabilístico.

La muestra estuvo conformada por 191 personas de edades entre 55 y 87 años, 58.6 $\%$ mujeres y $41.4 \%$ hombres que habitan en el área metropolitana [zona de la capital del país] y que participaban en los programas educativos dirigidos para personas mayores de 50 años y más, en universidades públicas de la Gran Área Metropolitana. Con este tamaño de muestra y los resultados obtenidos de las técnicas estadísticas utilizadas se obtuvo un poder estadístico de 0.99 y un tamaño del efecto de 0.25. El intervalo de edad fue amplio para captar la mayor variabilidad posible en el 
análisis, pero dentro de los rangos considerados internacionalmente como definitorios de la vejez (55 años en adelante). El rango de edad permitió también contar con mayor diversidad en las etapas de la vejez, aspecto que ha sido muy discutido como carente en la indagación del envejecimiento con éxito (Baltes \& Smith, 2003; Cho et al., 2015), a pesar de que la mayoría de la muestra estudiada se ubicó en una media de 68.5 años de edad $(D E=6.9)$.

Entre las personas participantes del estudio, el 84 \% había alcanzado la escolaridad secundaria y universitaria, con una media de 13.3 años de estudio formal $(D E=4.7)$, el $82 \%$ dijo estar jubilado y un $74 \%$, recibir pensión de alguno de los regímenes nacionales. Finalmente, el 65.4 $\%$ informó tener un ingreso aproximado de $\$ 400$ a $\$ 1.100$ mensuales y un $26.1 \%$ entre $\$ 1.200$ y mayor a $\$ 2.000$ mensuales. Todos acordaron participar de forma voluntaria, mediante la firma de un consentimiento informado, tomando en cuenta los reglamentos de ética de investigación en seres humanos de la Universidad de Costa Rica.

\section{Variables e instrumentos}

En la Tabla 1, se presentan los instrumentos de medición utilizados (para mayor información, véanse, Fernández-Ballesteros et al., 2004; Blanco-Molina \& Salazar-Villanea, 2014a, 2014b, 2014c).

\section{TABLA 1}

Instrumentos utilizados según variables en el modelo de análisis

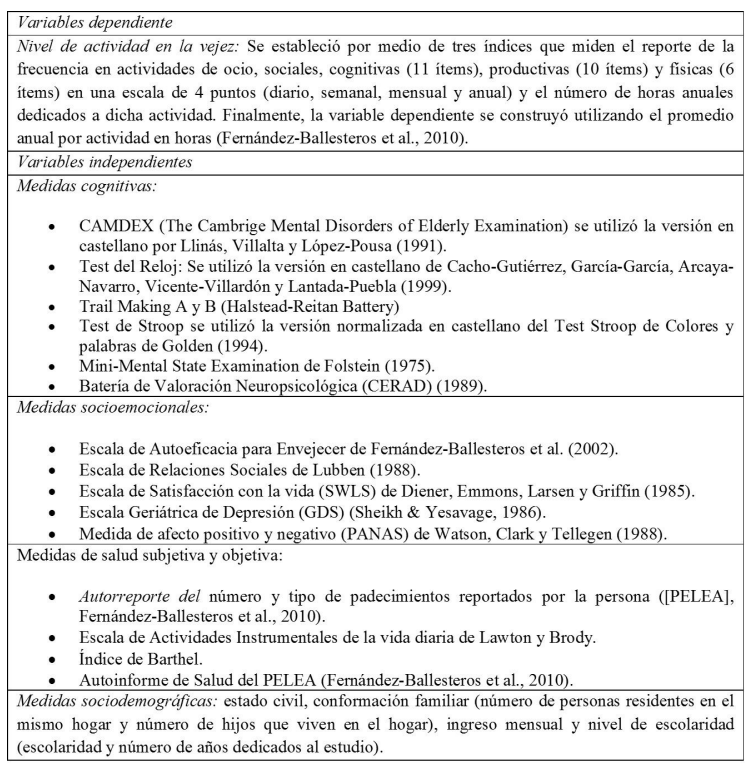

Fuente: elaboración propia

Procedimientos y análisis estadístico

Las entrevistas se realizaron de forma individual cara a cara, con una duración aproximada de 2.5 horas por persona, en espacios adecuados y cómodos para la aplicación de cada instrumento.

Cada variable independiente correspondió a los puntajes brutos en el caso de test o pruebas cognitivas, y/o a un puntaje escalar, mediante las medias, en el test de actitudes y/o índices utilizados. En las variables no continuas, se procedió a su debida transformación en variables dummy.

Se realizó un análisis de componentes principales con rotación VARIMAX para maximizar la varianza de las medidas (Martínez, Hernández, \& Hernández, 2006), y asî poder agrupar las variables socioemocionales y cognitivas que han sido identificadas como predictoras del envejecimiento con éxito en la literatura clásica y en la más actual (Baltes \& Baltes, 1990; Fernández-Ballesteros et al., 2002; Freund et al., 2009; Jopp \& Smith, 2006; Parslow et al., 2011).

El análisis factorial se realizó con la intención de definir el mejor grado de identificación de la 
multidimensionalidad con mejor ajuste para la predicción del modelo del envejecimiento con éxito, que en este estudio se definió a partir del nivel de participación social y física; para esto, se utilizó el modelo de componentes principales (CP) (Floyd \& Widaman, 1995). Como criterios de ajuste del análisis, se tuvo en cuenta que los valores característicos fueran mayores a 1 , como criterio para identificar factores significativos de agrupación de las variables. Los componentes identificados se construyeron con las variables con cargas superiores a 4. Además, se usó el criterio de Kaiser-Meyer-Olkin (KMO) superior a 0.8, como medida de la interrelación entre los factores (variables que lo componen, véase Tabla 1) y como indicador de consistencia interna factorial. Con el CP, se agruparon las variables independientes en dos grandes medidas globales latentes: a) recursos personales cognitivos y b) recursos socioemocionales.

Se optó por este análisis por sus cualidades como técnica estadística de síntesis de la información para la reducción de las dimensiones (número de variables), en el análisis de interdependencias o interrelaciones. Es decir, orientados por la pregunta de investigación, esta técnica permite observar la relación de los factores con las variables iniciales y en coherencia con la teoría que subyace. Esta elección es pertinente según los antecedentes de estudios similares en temas de salud pública y envejecimiento, que justamente trabajan con múltiples indicadores y variables para describir fenómenos complejos. Así, por ejemplo, este tipo de análisis se ha utilizado previamente en investigaciones asociados a la calidad de vida o a condiciones de salud y vulnerabilidad en población adulta mayor, estudios geográficos sobre envejecimiento y/o análisis de conductas discriminatorias (Cardona-Arango \& EstradaRestrepo, 2002; Cardona, Estrada, \& Agudelo, 2006; Castellano-Fuentes \& de Miguel-Negredo, 2011; Sánchez-González, 2009; Rojo Pérez \& Fernández-Matoralas, 2002).

Se calcularon también análisis de correlación r de Pearson, para el caso de las variables continuas y Rho de Spearman cuando se compararon variables continuas con ordinales. También se llevó a cabo un análisis de regresión lineal múltiple, mediante el método jerárquico, para identificar los mejores predictores de los diversos niveles de actividad, medidos como criterio de envejecimiento con éxito. Todos los análisis se hicieron mediante el programa SPSS para Windows (v 19.0).

\section{Resultados}

Perfil de participación en el estilo de vida activo: tipo y frecuencia de actividades reportadas

En primer lugar, se describen los datos de la variable dependiente (criterio de envejecimiento con éxito) del modelo, definida como el número de horas anuales dedicadas a la realización de actividades sociales, de ocio, cognitivas, productivas-instrumentales y físicas (Tabla 2).

\section{TABLA 2}

Descriptivos según el tipo de actividad en horas al año y descripción de la actividad física

\begin{tabular}{|c|c|c|c|c|c|}
\hline Variable & $n$ & $m$ & $D E$ & $\begin{array}{l}\text { Puntaje } \\
\text { minimo }\end{array}$ & $\begin{array}{l}\text { Puntaje } \\
\text { máximo }\end{array}$ \\
\hline Número de horas anuales dedicadas a hacer ejercicio físico & 191 & 247.5 & 275 & 0 & 2190 \\
\hline Descripción de la actividad fisica * & 191 & 4.3 & 1.46 & 1 & 7 \\
\hline $\begin{array}{l}\text { Número de horas anuales dedicadas a hacer actividades } \\
\text { productivas }\end{array}$ & 191 & 2379.7 & 2179 & 0 & 17220 \\
\hline $\begin{array}{l}\text { Número de horas anuales dedicadas a hacer actividades de } \\
\text { ocio }\end{array}$ & 191 & 2166.7 & 1547.5 & 0 & 8158 \\
\hline $\begin{array}{l}\text { Número de horas anuales dedicadas a hacer actividades } \\
\text { sociales }\end{array}$ & 191 & 644.4 & 616.9 & 0 & 3551 \\
\hline $\begin{array}{l}\text { Número de horas anuales dedicadas a hacer actividades } \\
\text { cognitivas }\end{array}$ & 191 & 448.8 & 615.3 & 0 & 5475 \\
\hline $\begin{array}{l}\text { Nota: * En la escala } \\
\text { de la actividad física, a } \\
\text { mayor intensidad de la } \\
\text { Fuente: elaboraci }\end{array}$ & & propi & d fí & & \\
\hline
\end{tabular}

Las personas participantes del estudio dedicaban de 2 a 3 horas por semana en promedio a este tipo de actividades y un $81.2 \%$ dijo hacer ejercicio diario y/o semanalmente. Las prácticas de tipo productivas fueron definidas como aquellas dirigidas a establecer o mantener la productividad no económica en la vida de las personas estudiadas, cómo, por ejemplo: tareas del hogar, voluntariado, cuidado de personas enfermas o niños, compras y trámites personales, manualidades, entre otras. Es importante mencionar que internacionalmente -parámetro europeo y español- (FernándezBallesteros, 2004; Fernández-Ballesteros et al., 
2010; Potočnik \& Sonnentag, 2013) este tipo de acciones se han clasificado como productivas, aunque representan principalmente características que reflejan funcionalidad instrumental (actividades de la vida diaria), específicamente. Con respecto a este tipo de actividades, las personas estudiadas reportan dedicar al año en promedio 2379.7 horas o 45.76 horas semanales.

En cuanto a actividades de ocio como ver la televisión, escuchar la radio, jugar en solitario o en compañía e ir a espectáculos, las personas dedican al año en promedio 2166.7 horas. Principalmente, estas horas son dedicadas a ver la televisión o a escuchar radio, es decir, aproximadamente 6 horas al día; $88 \%$ de la muestra dijo ver la televisión a diario y un $68 \%$ escucha radio diariamente, siendo las actividades en las cuales dedican más tiempo, luego de las de tipo productivas (instrumentales).

Hay que tomar en cuenta que aunque se clasificaron como actividades de ocio para el presente estudio, se carece de la información detallada para especificar sus peculiaridades. Por ejemplo, en cuanto a la dedicación de muchas horas a ver la televisión, no se recogió la información sobre el tipo de programas, sus contenidos ni su calidad, aspectos que deberán ser tomados en cuenta como una limitación del estudio y una oportunidad de indagación futura. A los actos de tipo social, como visitar a amigos, hacer excursiones, ir a la Iglesia y hacer viajes, las personas dedican al año 644.4 horas, aproximadamente 1.7 horas al día. Finalmente, a las actividades de tipo cognitivo (específicamente, lectura de libros y periódicos), las personas reportaron dedicar anualmente 448.8 horas en promedio, o en otras palabras, 1.2 horas al día.

Características socioemocionales de la muestra estudiada: el papel del afecto positivo

Las características socioemocionales reportadas muestran medias altas en las escalas que evaluaban aspectos positivos del estado de ánimo, satisfacción con la vida, percepción de redes de apoyo y autoeficacia. Por otro lado, se evidenciaron bajos puntajes en medidas de afecto negativo o evidencia de estados depresivos, lo que es congruente con los puntajes altos de las escalas de valencia positiva.

En la Tabla 3, se detallan los puntajes de las escalas y las medidas de consistencia interna, según el indicador alpha de Cronbach.

\section{TABLA 3}

Descriptivos variables medidas según escalas e indice de confiabilidad interna

\begin{tabular}{|c|c|c|c|c|c|c|}
\hline Variable & $n$ & $m$ & $D E$ & $\begin{array}{l}\text { Puntaje } \\
\text { mínimo }\end{array}$ & $\begin{array}{l}\text { Puntaje } \\
\text { máximo }\end{array}$ & $\begin{array}{l}\text { alpha } \\
\text { Cronbach }\end{array}$ \\
\hline Panas Negativo (estado de ánimo)* & 191 & 22.2 & 8 & 10 & 45 & 0.87 \\
\hline $\begin{array}{l}\text { Panas Positivo (estado de ánimo) } \\
\text { Escala Geriátrica de Depresión }\end{array}$ & 191 & 40.5 & 6.4 & 18 & 50 & 0.83 \\
\hline GDS(estado de ánimo)* & 191 & 5.4 & 5.1 & 0 & 25 & 0.81 \\
\hline Satisfacción con la vida & 191 & 28.1 & 6 & 6 & 35 & 0.87 \\
\hline Autoeficacia para envejecer & 191 & 40.3 & 6 & 19 & 50 & 0.87 \\
\hline $\begin{array}{l}\text { Control percibido } \Delta \\
\text { Red apovo familia }\end{array}$ & $\begin{array}{l}189 \\
188\end{array}$ & $\begin{array}{l}2.3 \\
13.7\end{array}$ & $\begin{array}{l}3.3 \\
4\end{array}$ & $\begin{array}{l}1 \\
3\end{array}$ & 7 & - \\
\hline Red de apoyo amistades & 191 & 14.4 & 17.5 & 0 & 204 & - \\
\hline Red de apoyo confianza & 191 & 23.4 & 5.3 & 7 & 30 & 0.63 \\
\hline $\begin{array}{l}\text { Tamaño del hogar (número de } \\
\text { personas que viven en el hogar) } \\
\text { Número de hijos que viven en el }\end{array}$ & 191 & 2.6 & 1.4 & 1 & 8 & - \\
\hline hogar & 161 & 1.4 & 1.3 & 0 & 6 & - \\
\hline
\end{tabular}

Nota: * Puntajes bajos evidencian bajo riesgo a sufrir o tener estados depresivos y presencia de emociones negativas. $\Delta$ Bajos puntajes evidencian mayor presencia de control interno y altos puntajes presencia de control externo. Fuente: elaboración propia.

La mayoría de las personas reporta mayor presencia de emociones positivas, creen tener las capacidades necesarias para superar las dificultades de la vida y de la vejez y se consideran personas satisfechas con su situación actual y pasada. Cabe señalar que se registraron puntuaciones medias y altas con respecto a las escalas que evaluaban percepción de apoyo en tres aspectos: a) apoyo familiar (con las personas con las que viven y familia extensa), b) amistades y c) ellas mismas como sujetos para otorgar apoyo a las demás personas (confianza). La mayoría de participantes conviven con su pareja e hijos, lo que habla de la conformación familiar tradicional en el esquema de vida de las personas adultas mayores residentes en Costa Rica (Fernández \& Robles, 2006; Rosero-Bixby \& Dow, 2009). 
Perfil de rendimiento cognitivo: relación con la funcionalidad e independencia

Las características cognitivas estudiadas también presentan puntajes altos en los test de ejecución que evaluaron los cuatro constructos principales: medidas de screening (cribaje o despistaje), memoria, lenguaje y funciones ejecutivas. Perfilar el rendimiento cognitivo es relevante en esta investigación por su relación directa con la funcionalidad y la independencia que caracteriza a la muestra seleccionada (Tabla 4).

\section{TABLA 4}

Descriptivos de las característica cognitivas en la muestra estudiada

\begin{tabular}{|c|c|c|c|c|c|}
\hline Variables y escalas & $n$ & $M$ & $D E$ & $\begin{array}{l}\text { Puntaje } \\
\text { mínimo }\end{array}$ & $\begin{array}{l}\text { Puntaje } \\
\text { máximo }\end{array}$ \\
\hline \multicolumn{6}{|l|}{ Screening o despistaje } \\
\hline Mini-Mental State Examination (MMSE) & 190 & 28.3 & 2 & 19 & 30 \\
\hline \multicolumn{6}{|l|}{ Funciones Ejecutivas } \\
\hline Stroop & 191 & 4.3 & 7.9 & -17.5 & 34.7 \\
\hline Trail Making A & 190 & 2 & 1 & 1 & 6 \\
\hline Trail Making B & 190 & 2.5 & 1.7 & 1 & 6 \\
\hline CERAD digitos directos & 190 & 5.3 & 1.1 & 3 & 9 \\
\hline CERAD digitos inversos & 190 & 3.5 & 1 & 2 & 6 \\
\hline \multicolumn{6}{|l|}{ Lenguaje } \\
\hline CERAD fluidez verbal & 191 & 19.5 & 4.6 & 9 & 31 \\
\hline CAMCOG lenguaje comprensión & 191 & 8.9 & 0.4 & 7 & 9 \\
\hline CAMCOG lenguaje expresión & 191 & 17.7 & 2.1 & 12 & 24 \\
\hline \multicolumn{6}{|l|}{ Memoria } \\
\hline CAMCOG memoria remota & 191 & 5.8 & 0.6 & 3 & 6 \\
\hline CAMCOG memoria reciente & 191 & 3.6 & 0.6 & 1 & 4 \\
\hline CAMCOG memoria aprendizaje & 191 & 12.9 & 2.4 & 5 & 17 \\
\hline
\end{tabular}

Fuente: elaboración propia

En la muestra, cuyo rendimiento intelectual global se mantiene preservado, las escalas de screening confirmaron la ausencia de indicadores de sospecha de deterioro cognitivo. Las medidas de memoria evidenciaron un rendimiento esperable en la mayoría de indicadores de los procesos de codificación y recuperación de información de los estímulos de los test de ejecución. De forma general, los resultados ponen de manifiesto que existe en la muestra estudiada una funcionalidad cognitiva y ausencia de deterioro cognitivo. Debe resaltarse también que el desempeño en las pruebas que evaluaban función ejecutiva estuvo caracterizado por puntajes medios, lo cual es coherente con las evidencias del cambio cognitivo en la vejez (es esperable que los principales declives en el desempeño se presenten en las funciones de memoria de trabajo y la velocidad de procesamiento de la información). Ahora, resulta relevante decir que, aunque la muestra en general no presenta un desempeño cognitivo con puntajes muy altos en relación con muestras de países desarrollados (Schaie, 2005), estas capacidades les resultan adecuadas para adaptarse a las demandas de su vida cotidiana, en un entorno que posiblemente no sea muy demandante cognitivamente, aspecto que deberá ser indagado con mayor detenimiento en otro momento.

Percepción subjetiva positiva del estado de salud y su contraste con el reporte de condiciones patológicas: aspectos de interés en la muestra estudiada

Las personas en su mayoría (77 \%) no reportaron ningún tipo de padecimiento que dificultara de alguna manera la actividad cotidiana. Sin embargo, la mayoría (55 \%) informó tener algún problema médico diagnosticado, principalmente patologías como Diabetes Mellitus, hipertensión arterial, arterioesclerosis, patologías relacionadas con la glándula tiroides, problemas musculares y óseos, además de consumir por esos padecimientos, en promedio $3.1(\mathrm{DE}=2.5)$ medicamentos al día.

A pesar de estas condiciones, la mayoría de la muestra (considerando las medidas de funcionalidad en actividades de la vida cotidiana e instrumental) está constituida por personas funcionalmente independientes. Es interesante evidenciar que, aunque presentan algún tipo de patología, refieren que su salud es muy buena y que están mejor que otros de su misma edad y sexo, como lo evidencia la medida de salud subjetiva (Tabla 5).

El 70 \% considera que su visión y audición se encuentra en un estado bueno y muy bueno y el $73.3 \%$ manifiesta no fumar o nunca haberlo hecho. Con respecto a la ingesta de bebidas alcohólicas, el consumo de cerveza, vino y licor puro, la mayoría no consume este tipo de bebidas ( $81.8 \%$ ) y se considera abstemia (los que reportan consumo, principalmente lo hacen una vez al mes y en ocasiones especiales). 


\section{TABLA 5}

Descripción, características de salud según frecuencia y porcentaje en la muestra

\begin{tabular}{llllllll}
\hline & & & & & Puntaje & Puntaje \\
Escala & $N$ & $m$ & $D E$ & mínimo & máximo \\
\hline Escala Lawton y Brody (Funcionalidad actividades instrumentales) & 189 & 7.97 & 0.17 & 7 & 8 \\
İndice de Barthel (Funcionalidad actividades básicas) & 189 & 99.6 & 2.52 & 70 & 100 \\
Salud subjetiva percibida & 191 & 36 & 5.3 & 16 & 45 \\
\hline
\end{tabular}

Fuente: elaboración propia

Predictores del perfil de participación y el estilo de vida activo: el papel de las variables socioemocionales, cognitivas, de salud y sociodemográficas

Es importante considerar en estos resultados la limitante del poder estadístico y el tamaño del efecto alcanzado, aunque bueno, al ser una muestra no probabilística y muy homogénea en sus características. Se considera, sin embargo, que el estudio funge como un primer acercamiento descriptivo al fenómeno en el contexto costarricense, que aporta importantes contribuciones metodológicas para futuras propuestas.

Tomando en cuenta lo dicho, el análisis correlacional evidencia que el promedio anual en actividades de ocio se relacionó positivamente con una medida que agrupa a las escalas de evaluación de funciones ejecutivas, construidas mediante la técnica de componentes principales (15.8\% varianza explicada, 1.8 valor característico) y con una medida de salud relacionada con la percepción del estado del oído (Tabla 6).

Por su parte, las actividades de tipo social correlacionaron positivamente con variables sociodemográficas, como nivel educativo, ingreso y número de hijos, residiendo en el hogar. Se evidenciaron también relaciones positivas con la medida global de características socioemocionales, construida mediante la técnica de componentes principales (42\% varianza explicada, 2.9 valor característico), que agrupa las medidas de estado emocional, eficacia y control percibido y redes de apoyo.

También se manifestó la presencia de relaciones positivas con las medidas de screening
(42\% de la varianza explicada, 1.8 valor característico), la presencia de patologías y la percepción reportada de la condición del oído y la visión (Tabla 7, para mayor detalle del análisis $\mathrm{CP}$.

La actividad cognitiva se relacionó positivamente únicamente con el ingreso registrado y una variable construida a partir de un análisis por componentes principales que agrupó a las medidas de lenguaje (56 \% varianza explicada, 1.8 valor característico).

TABLA 6

Correlaciones de $r$ Pearson entre los tipos de actividad y las variables medidas

\begin{tabular}{|c|c|c|c|c|c|}
\hline $\begin{array}{l}\text { Variables } \\
\end{array}$ & Ocio & Sociales & Cognitivas & Fisicias & Productivas \\
\hline $\begin{array}{l}\text { Edad } \\
\text { Ead }\end{array}$ & & & & $-0.15^{*}$ & \\
\hline Años de estudio & & $0.27^{* * *}$ & & $0.19^{\circ *}$ & $-0.19^{* * *}$ \\
\hline Ingreso & - & $0.22^{4 *}$ & $0.16^{\circ}$ & $0.16^{\circ}$ & $-0.26^{* *}$ \\
\hline $\begin{array}{l}\text { \# de hijos } \\
\text { Tamaño del hogarar }\end{array}$ & : & $0.22^{* *}$ & & & \\
\hline$\frac{\text { Tamanà del hogar }}{\text { Socio-emocionales }}$ & & $031^{* *}$ & & $\frac{0.17^{*}}{0.17^{*}}$ & $0.19^{* * *}$ \\
\hline Screening & & $0.18^{*}$ & & & 0.10 \\
\hline Funciones ejecutivas" & $0.16^{*}$ & & & $0.2^{ \pm *}$ & \\
\hline Memoria* & & & & - & $-0.15^{*}$ \\
\hline$\frac{\text { Lenguajaje }}{\text { Salud subetiva }}$ & & & & & \\
\hline $\begin{array}{l}\text { Salud unjbetiva } \\
\text { Presencia patologias }\end{array}$ & & & & $0.017^{\circ}$ & \\
\hline $\begin{array}{l}\text { Presencua patologias } \\
\text { Estado del oido }\end{array}$ & 0 & 0.000 & & & \\
\hline Estado de la visión & 0.19 & $0.14^{*}$ & & & \\
\hline consumo tabaco & & & & & $-0.2^{* * *}$ \\
\hline
\end{tabular}

Nota: $* p<0.05 * * p<0.01$. Variables globales agrupadas por la técnica de CP: a)

Socioemocionales: GDS, PANAS, autoeficacia, satisfacción, control y red apoyo social. $42 \%$ varianza explicada, 2.9 valor característico; b) Screening: Mini-Mental, TRO, CAMCOG atención y concentración, orientación, atención y cálculo. $42.7 \%$ varianza explicada, 2.1 valor característico; c) Funciones ejecutivas: STROOP y Dígitos directos e inversos, 15.8\% varianza explicada, 1.8 valor característico; d) Memoria: Memoria remota, reciente y aprendizaje. $15.6 \%$ varianza explicada, 1.2 valor característico y e) Lenguaje: Fluidez, expresión verbal y comprensión verbal 56\% varianza explicada, 1.8 valor característico. Fuente: elaboración propia

La actividad física presentó correlaciones negativas con la edad reportada y relaciones positivas con variables como nivel educativo, ingreso, número de hijos, medida global resultante del agrupamiento de las escalas socioemocionales y funciones ejecutivas. Por último, presentaron relaciones positivas con características de salud como presencia de patologías y percepción de salud subjetiva.

Finalmente, las actividades productivas/ instrumentales correlacionaron negativamente con el nivel educativo, ingreso reportado, 
medida factorial resultante de variables socioemocionales, variable global de memoria (15.6\% varianza explicada, 1.2 valor característico) y con el consumo de tabaco. Presentaron, sin embargo, correlaciones positivas con el tamaño del hogar.

Se presentan a continuación, en la Tabla 7, las cargas factoriales asociadas a cada factor que resultó del análisis factorial por medio de la técnica de CP. El modelo resultante identifica un solo factor predictor que se describe en la siguiente tabla.

\section{TABLA 7}

Análisis Factorial. Método CP de las variables socioemocionales y cognitivas medidas

\begin{tabular}{lll}
\hline Variables & Carga Factorial & $\mathbf{h}^{2}$ \\
\hline Predicotores Socioemocionales & & \\
Red Social & 0.47 & 0.22 \\
PANAS Positivo & 0.64 & 0.41 \\
Autoeficacia & 0.72 & 0.52 \\
Control Percibido & -0.46 & 0.21 \\
Satisfacción con la vida & 0.73 & 0.53 \\
Depresión (GDS-Yesavage) & -0.79 & 0.62 \\
PANAS Negativo & -0.67 & 0.45 \\
\% varianza acumulada explicada & & 42 \\
\hline Predicotores cognitivos & & \\
Screening & 0.83 & 0.69 \\
Mini-Mental & 0.64 & 0.41 \\
Test del Reloj & 0.72 & 0.52 \\
CAMCOG Orientación y cálculo & & 42.7 \\
\% varianza acumulada explicada & & \\
\hline Funciones ejecutivas & 0.65 & 0.42 \\
Stroop & 0.46 & 0.42 \\
Digitos WAIS & & 15.8 \\
\% varianza acumulada explicada & & \\
\hline Memoria & & 0.61 \\
CAMCOG Memoria inmediata & 0.75 & 0.57 \\
CAMCOG Memoria remota & 0.75 & 15.6 \\
\% varianza acumulada explicada & & 0.75 \\
\hline Lenguaje & & 0.77 \\
CAMCOG Fluidez y expresión verbal & 0.87 & 56 \\
CAMCOG comprensión verbal & 0.88 & \\
\% varianza acumulada explicada & & \\
& & \\
\hline & & \\
\hline
\end{tabular}

Fuente: elaboración propia

La introducción de las variables en el análisis de regresión lineal se basó en los antecedentes empíricos que han identificado a los mejores predictores del envejecimiento con éxito de la siguiente forma: (a) las medidas sociodemográficas (Gestorf et al., 2008; Steverink et al., 1998), (b) las medidas socioemocionales (Baltes \& Lang, 1997; Kahana et al., 2002), (c) las medidas cognitivas (Albert et al., 1995; Strawbrige, Cohem, Schema, \& Kaplan, 1996) y (d) las medidas de salud (Haveman-Nies et al., 2003; Kahana \& Kahana, 2001).

\section{TABLA 8}

Análisis de regresión lineal (Enter) para las variables que mejor predicen la actividad en la muestra

\begin{tabular}{|c|c|c|c|c|}
\hline Mejores modelos de predicción seguin tipo de actividad & $B$ & $R^{2}$ & $\Delta R^{2}$ & F Regresión \\
\hline Actividad social $^{4}$ & & 0.24 & 0.04 & $F_{(5,138) 2.6 * *}$ \\
\hline 1. Nủmero de hijos que viven en el hogar & $0.19^{*}$ & & & \\
\hline 2. Socioemocionales & $0.24 * *$ & & & \\
\hline 3. Estado del oído & $0.16^{*}$ & & & \\
\hline Actividad cognitivat & & 0.37 & 0.15 & $F_{(41,127) 1.9 * *}$ \\
\hline 1. Ingreso mensual & $0.25 * *$ & & & \\
\hline 2. PANAS Positivo & $0.33 * *$ & & & \\
\hline 4.Consumo tabaco & $-0.43^{*}$ & & & \\
\hline 5.Trail Making A & $0.22 *$ & & & \\
\hline 6.CAMCOG Atención y cálculo & $-19^{*}$ & & & \\
\hline Actividad de ocio ${ }^{A}$ & & 0.25 & 0.07 & $F_{(34,147)} 1.1$ \\
\hline 1. CAMCOG Pensamiento abstracto & $0.28^{*}$ & & & \\
\hline 2. PANAS Positivo & $0.21^{*}$ & & & \\
\hline 3. Audición & $0.21^{*}$ & & & \\
\hline Actividad productiva/instrumental $^{A}$ & & 0.25 & 0.04 & $F_{(19,128) 2.2 * *}$ \\
\hline 1.Edad cronológica & $-0.3 * *$ & & & \\
\hline 2. Nivel educativo & $-0.2 *$ & & & \\
\hline 3.Tamaño del hogar & $0.19^{*}$ & & & \\
\hline 4.Socioemocionales & $-0.21^{*}$ & & & \\
\hline 5. Consumo de tabaco & $-0.4^{* *}$ & & & \\
\hline Actividad fisica $^{4}$ & & 0.26 & 0.06 & $F_{(23,176) 2,4^{* *}}$ \\
\hline 1. Edad cronológica & $-0.2^{*}$ & & & \\
\hline 2. Tamaño del hogar & $0.27 * *$ & & & \\
\hline 3. Cantidad consumo de alcohol & $-0.29 * *$ & & & \\
\hline
\end{tabular}

Nota: $* p<0.05 * * p<0.01 . \Delta 1$. Se incluyen en el siguiente orden las variables en los modelos de predicción: 1. Características sociodemográficas, 2. Características socioemocionales, 3. Características cognitivas y 4 . Características de salud.

Solamente en el modelo de predicción de las actividades cognitivas, el orden varió incluyendo a las características cognitivas de último. Fuente: elaboración propia

Se puede observar en la Tabla 8 que la dedicación a la participación en grupos sociales y contacto con amistades (actividad social) fue predicha en un $24 \%$ de la varianza explicada, principalmente por la cantidad de hijos residiendo en el mismo hogar, por las medidas socioemocionales (estado emocional, autoeficacia, control percibido y satisfacción con la vida) y, finalmente, por la percepción de contar con una buena capacidad auditiva.

Las actividades de tipo cognitivas, específicamente las horas dedicadas a la lectura, con un $37 \%$ de la varianza explicada fueron predichas por el ingreso reportado, un indicador socioemocional, como la presencia de emociones positivas (PANAS), ausencia de consumo de tabaco y mayor número de personas residiendo en el hogar. Las actividades de ocio, donde la predicción resultó no significativa, principalmente ver la televisión y oír la radio, no evidencian relación o predicción alta con las variables medidas.

Resulta interesante plantearse a futuro cuáles variables son las que predicen este tipo de actividades en la vejez. A pesar de 
ello, se reconoce un $25 \%$ de la varianza explicada por criterios cognitivos como funciones ejecutivas, socioemocionales como la presencia de emociones positivas (PANAS) y la percepción de una buena condición de la audición, que resultan de interés para el análisis.

Continuando con los resultados, se puede observar que las actividades productivas o instrumentales (hacer tareas el hogar, compras, mandados, etc.) fueron predichas en un 25 $\%$ de la varianza, por tener menor edad, menor consumo de tabaco y menor nivel educativo, vivir con más personas dentro del hogar y con indicadores negativos de criterios socioemocionales. Esto podría indicar, en la muestra, que la prioridad de estas actividades sobre otras de tipo social, físico, de ocio o cognitivo, se relaciona con un estado emocional negativo.

Por último, la actividad física fue predicha en un $26 \%$ de la varianza, debido al menor consumo de alcohol, a un mayor tamaño del hogar o más personas residiendo en el mismo hogar y a tener menos edad, es decir, conforme aumentaba la edad en la muestra, menor era el número de horas dedicadas al ejercicio físico. A continuación, se discutirán los hallazgos, tomando en cuenta la teoría del envejecimiento con éxito y los antecedentes empíricos sobre el fenómeno en distintos contextos.

\section{Discusión}

Orientada por la meta de estudiar y comprender las características de las personas adultas mayores con altos niveles de actividad, la presente investigación sirve de base empírica para la descripción de los factores predominantemente asociados a un estilo de vida funcional, independiente y satisfactorio, en la vejez de un grupo de personas residentes en Costa Rica.

Como se pudo observar en los resultados, estas personas presentaron indicadores socioemocionales positivos, como altas creencias de autoeficacia, mayor precepción de control interno, satisfacción con la vida actual, satisfacción con redes de apoyo emocional en el ámbito familiar y de amistades y ausencia de indicadores de estados depresivos. Estudios similares a nivel nacional e internacional (Blanco-Molina, 2007, 2010; FernándezBallesteros et al., 2010) han corroborado la relación entre grados altos de actividad y la presencia de estabilidad emocional, de salud subjetiva y desempeño cognitivo como criterios asociados al compromiso activo de participar en la sociedad (Baltes \& Lang, 1997; Ford et al., 2000; Glei et al., 2005; Kahana et al., 2012; Potočnik \& Sonnentag, 2013; Seeman et al., 1996).

Sin embargo, en la muestra estudiada se evidencian diferencias importantes entre los factores que predicen los distintos tipos de actividad, aspecto relevante para el desarrollo de programas que promuevan estilos de vida saludables durante la vejez.

En coherencia con el hallazgo internacional sobre el mantenimiento de las capacidades cognitivas como predictor de la adaptación, la participación y la salud en la vejez (Dockree et al., 2015; Fratiglioni, Paillard-Borg, \& Winbland, 2004; Hogan, Mata, \& Carstensen, 2013; Krueger et al., 2009; Vásquez et al., 2014), la muestra descrita coincide con los criterios de un rendimiento intelectual global preservado y una percepción positiva de salud, a pesar de la presencia de indicadores de medidas de rendimiento cognitivo menores a otras muestras descritas internacionalmente y presencia de mayores patologías reportadas.

Los datos mostraron que las personas con niveles educativos altos dedican mayor tiempo a actividades de ocio y de tipo social, siendo las actividades de tipo cognitivas, físicas u orientadas al logro personal, las que menos definen su perfil de participación cotidiana. Esto difiere de lo advertido internacionalmente y da cuenta quizás de una particularidad cultural.

Considerando que para el envejecimiento con éxito el criterio no es la cantidad de tiempo que se dedique a una actividad, sino si esta es relevante a nivel personal y permite la optimización y compensación de metas (Baltes \& Baltes, 1990), estos resultados permitirían potenciar el estudio del fenómeno de envejecimiento en contextos 
latinoamericanos, donde la participación social y las redes de apoyo se perciban mayormente relacionadas con el bienestar y la estabilidad (en contraste con muestras donde la participación está predominantemente orientada al logro, por ejemplo). Ahora bien, resulta necesario indagar hacia el futuro cómo son estas redes sociales y de apoyo, con el interés de comprender la dinámica de interacción y el entorno en el que viven estas personas que reportan altos niveles de actividad social en su vejez.

Otro dato interesante por discutir es la evidencia de las relaciones positivas del nivel de actividad con el ingreso declarado y el grado educativo, pues, como se ha demostrado ampliamente en otros estudios (Gestorf et al., 2008; Parslow et al., 2011), las poblaciones económica y socialmente favorecidas son las más propensas a reportar en la vejez mayor calidad de vida y satisfacción por la condición actual, mejor salud y condiciones cognitivas superiores.

Sobre este aspecto, es relevante acotar que la muestra no es representativa de la población mayor en Costa Rica, al ser personas que cumplen características relativas a la descripción de "superactivos", específicos de pequeños grupos de personas mayores que cumplen con los criterios del envejecimiento con éxito y que responden al principal propósito del presente estudio (Fernández-Ballesteros et al., 2011; Johnson et al., 2015).

Con los hallazgos, se cree pertinente plantear que podrían existir matices o perfiles en el modelo del envejecimiento con éxito en lugar de considerarlo una variable categórica (Fernández-Ballesteros et al., 2010), y para ello sería pertinente estudiar muestras de mayor heterogeneidad y tamaño. Asimismo, para estudios futuros es importante replantearse una mejor clasificación de los tipos de actividades indagadas, pues resulta difícil establecer una línea que separe a las actividades que son estrictamente de ocio, de aquellas sociales o que sean estimulantes cognitivamente, ya que entre ellas comparten características que las relacionan (Gallacher, Bayer, \& Ben-Shlomo, 2005).

Ante el fenómeno multifactorial del envejecimiento con éxito, que trasciende la buena salud física como criterio clásico (Rowe \& Khan, 1997), esta investigación aporta datos descriptivos del nivel de participación que le permite a la muestra estudiada maximizar los recursos personales con el beneficio percibido del tiempo dedicado a la participación social y ocio (Kahana \& Kahana, 1996, 2001; Kahana et al., 2014). Así las personas parecen invertir tiempo, incrementando estas actividades como estrategia de optimización de los propios recursos (Freund et al., 2009; Kahana et al., 2012; Lang \& Carstensen, 1994; Mendes de León, 2005), en un proceso donde se trata de vivir lo mejor posible de acuerdo a su entorno y capacidades (FernándezBallesteros, 2009).

\section{Agradecimientos}

Esta investigación tuvo el apoyo de la Vicerrectoría de Investigación, Universidad de Costa Rica. Proyecto 723-B1-304. (VI-UCR). "Estudio de las características socioemocionales y cognitivas predictoras de la actividad en la vejez. Una aproximación al envejecimiento exitoso en el contexto costarricense".

\section{Referencias}

Albert, M., Jones, K., Savage, C., Berkman, L., Seeman, T., Blazer, D., \& Rowe, J. W. (1995). Predictors of cognitive change in older persons: MacArthur studies of successful aging. Psychology and Aging, 10(4), 578-589.

Baltes, P. B., \& Baltes, M. M. (1990). Psychological perspective on successful aging: The model of selective optimization and compensation. En P. B. Baltes \& M. M. Baltes (Eds.), Successful aging: Perspective from the behavioral sciences (pp. 1-34). Nueva York: Cambridge University Press.

Baltes, P. B., \& Smith, J. (2003). New frontiers in the future of aging: From successful aging of the young old to the dilemmas of the fourth age. Gerontology, 49(2), 123-135. 
Baltes, M. M., \& Carstensen, L. L. (1996). The process of successful ageing. Ageing and Society, 16, 397-422.

Baltes, M. M., \& Lang, F. R. (1997). Everyday functioning and successful aging: The impact of resources. Psychology and Aging, 12(3), 433-443.

Blanco-Molina, M. (2007). Factores psicosociales determinantes del envejecimiento activo o exitoso en personas adultas costarricenses (Tesis de maestría inédita). Universidad de Costa Rica, San José.

Blanco-Molina, M. (2010). Predictores psicosociales del envejecimiento activo: evidencias en una muestra de personas adultas mayores costarricenses. Anales en Gerontología, 6, 11-29.

Blanco-Molina, M., \& Salazar-Villanea, M. (2014a). Escala de Afectividad (PANAS) de Watson y Clark en Adultos Mayores, pp.72-76. En: "Compendio de Instrumentos de Medición IIP-2014". Smith-Castro, V. (Comp.). Serie: Cuadernos Metodológicos del IIP. http://iip.ucr.ac.cr/sites/default/files/cu adernosmetodologicos/cuamet6.PDF

Blanco-Molina, M., \& Salazar-Villanea, M. (2014b). Escala de Satisfacción con la Vida para adultos mayores de Emmons, Larsen y Griffin, pp. 227-231. En: "Compendio de Instrumentos de Medición IIP-2014". Smith-Castro, V. (Comp.). Serie: Cuadernos Metodológicos del IIP. Recuperado de: http://www.researchgate.net/publicatio n/269223147_Compendio_de_Instrument os_de_Medicin_IIP-2014

Blanco-Molina, M., \& Salazar-Villanea, M. (2014c). Escala de Depresión Geriátrica GDS de Yesavage, pp. 241-246. En: "Compendio de Instrumentos de Medición IIP-2014". Smith-Castro, V. (Comp.). Serie: Cuadernos Metodológicos del IIP. http://www.researchg ate.net/publication/269223147_Compendi o_de_Instrumentos_de_Medicin_IIP-2014

Bowling, A., \& Dieppe, P. (2005). ¿What is successful aging and who should define it? BJM, 331, 1548-1551. doi:10.1136/ bmj.331.7531.1548
Bowling, A., \& Iliffe, S. (2006). Which model of successful ageing should be used in epidemiological surveys? Baseline findings from a British survey of ageing. Age and Ageing, 35(6), 607-614.

Bowling, A., \& Iliffe, S. (2011). Psychological approach to successful ageing predicts future quality of life in older adults. Health and Quality of Life Outcomes, 9(13), 1-10. Recuperado de http://www.hqlo.com/conte $\mathrm{nt} / 9 / 1 / 13$

Bülow, M. H., \& Söderqvist, T. (2014). Successful ageing: A historical overview and critical analysis of a successful concept. Journal of Aging Studies, 31, 139-149.

Cacho, J., García-García, R., Arcaya, J., Vicente, J. L., \& Lantada, N. (1999). Una propuesta de aplicación y puntuación del test del reloj en la enfermedad de Alzheimer. Rev Neurol, 28(7), 648-655.

Cardona, D., Estrada, A., \& Agudelo, H. (2006). Calidad de vida y condiciones de salud de la población adulta mayor de Medellín. Biomédica, 26(2), 206-215.

Cardona Arango, M., \& Estrada Restrepo, A. (2002). Envejecer nos "toca" a todos caracterización de algunos componentes de calidad de vida y de condiciones de salud de la población adulta mayor (Informe de Proyecto). Medellín: Universidad de Antioquia, Facultad Nacional de Salud Pública. Recuperado de http://www.udea.edu.co/wps/wcm/conn ect/udea/abb2aa99-91f1-42f4-8258-5695a $5014 \mathrm{a} 73 /$

Castellano-Fuentes, C., \& de MiguelNegredo, A. (2011). Propiedades psicométricas del cuestionario Conductas Discriminatorias hacia la Vejez y el Envejecimiento (CONDUC-ENV): análisis del componente conductual de actitudes viejistas en ancianos. International Journal of Psychology and Psychological Therapy, 11(3), 359-375.

Cho, J., Martin, P., \& Poon, L. W. (2015). Successful aging and subjective well-being among oldest-old adults. Gerontologist, $55(1), 132-143$. 
Collin, C., Wade, D. T., Davies, S., \& Horne, V. (1988). The Barthel ADL Index: a reliability study. International disability studies, 10(2), 61-63.

Diener, E. D., Emmons, R. A., Larsen, R. J., \& Griffin, S. (1985). The satisfaction with life scale. Journal of personality assessment, 49(1), 71-75.

Dikmen, S. S., Heaton, R. K., Grant, I., \& Temkin, N. R. (1999). Test-retest reliability and practice effects of expanded Halstead-Reitan Neuropsychological Test Battery. Journal of the International Neuropsychological Society, 5(04), 346-356.

Dockree, P. M., Brennan, S., O’Sullivan, M., Robertson, I. H., \& O'Connell, R. G. (2015). Characterising neural signatures of successful aging: Electrophysiological correlates of preserved episodic memory in older age. Brain and Cognition, 97, 40-50. doi: 10.1016/j.bandc.2015.04.002

Eizenman, D., Nesselroade, J., Featherman, D., \& Rowe, J., (1997). Intraindividual variability in perceived control in an older sample: The MacArthur successful aging studies. Psychology and Aging, 12 (3), 489-502.

Fernández, X., \& Robles, A. (Coords.). (2008). I Informe estado de la situación de la personas adulta mayor en Costa Rica. San José, Costa Rica: Consejo Nacional de la Persona Adulta Mayor.

Fernández-Ballesteros, R. (2009). Envejecimiento activo: contribuciones de la psicología. Madrid: Pirámide.

Fernández-Ballesteros, R., Diez-Nicolás, J., Caprara, G. V., Barbaranelli, C., \& Bandura, A. (2002). Self-efficacy and collective efficacy: Structural relationships. Applied Psychology: An International Review, 51, 107-212.

Fernández-Ballesteros, R., Zamarrón, M. D., Díez-Nicolás, J., López-Bravo, M. D., Molina, M. Á., \& Schettini, R. (2011). Productivity in old age. Research on Aging, 33(2), 205-226.

Fernández-Ballesteros, R., Zamarrón, M. D., López, M. D., Molina, M., Díez, J., Montero, P., \& Schettini, R. (2010).
Envejecimiento con éxito: criterios y predictores. Psicothema, 22 (4), 641-647.

Fernández-Ballesteros, R., Zamarrón, M. D., Rudinger, G., Schroots, J. J., Hekkinnen E., Drusini, A., ... Rosenmayr, L. (2004). Assessing competence. The European Survey on Ageing Protocol. Gerontology, 50, 330-347.

Floyd, F. J., \& Widaman, K. F. (1995). Factor analysis in the development and refinement of clinical assessment instruments. Psychological Assessment, 7(3), 286-299.

Ford, A., Haug, M., Stange, K., Gaines, A., Noelker, L., \& Jones, P. (2000). Sustained personal autonomy: A measure of successful aging. Journal of Aging and Health, 12(4), 470-489.

Folstein, M. F., Robins, L. N., \& Helzer, J. E. (1983). The mini-mental state examination. Archives of general psychiatry, 40(7), 812-812.

Fratiglioni, L., Paillard-Borg, S., \& Winbland, B. (2004). An active and socially integrated lifestyles in late life might protect against dementia. Lancet Neurology, 3, 343-353.

Freund, A., \& Baltes, P. B. (1998). Selection, optimization, and compensation as strategies of life management: Correlations with subjective indicators of successful aging. Psychology and Aging, 13 (4), 531-543.

Freund, A. M., Nikitin, J., \& Ritter, J. O. (2009). Psychological consequences of longevity. Human Development, 52 (1), 1-37.

Gallacher, J., Bayer, A., \& Ben-Shlomo, Y. (2005). Commentary: Activity each day keeps dementia away\#does social interaction really preserve cognitive function? International Journal of Epidemiology, 34(4), 872-873. doi:10.1093/ ije/dyi107

Gestorf, D., Ram, N., Estabrook, R., Shupp, J., Wargner, G., \& Lindenberg, U. (2008). Life satisfaction shows terminal decline in old age: Longitudinal evidence from the German socio-economic panel study (SOEP). Developmental Psychology, 44(4), 1148-1159. 
Glei, D. A., Landau, D. A., Goldman, N., Chuang, Y. L., Rodriguez, G., \& Weinstein, M. (2005). Participating in social activities helps preserve cognitive function: An analysis of a longitudinal, population-based study of the elderly. International Journal of Epidemiology, 34(4), 864-871.

Golden, C. J. (1994). STROOP: Test de colores y palabras: Manual. Madrid: TEA ediciones.

Gureje, O., Oladeji, B. D., Abiona, T., \& Chatterji, S. (2014). Profile and determinants of successful aging in the Ibadan study of ageing. Journal of the American Geriatrics Society, 62 (5), 836-842. doi:10.1111/jgs. 12802

Havighurst, R. (1963). Successful aging. En R. H. Williams, C. Tibbitts \& W. Donahue (Eds.), Processes of aging (pp. 299-332). Nueva York: Atherton Press.

Haveman-Nies, A., de Groot, L., \& van Staveren, W. A. (2003). Dietary quality, lifestyle factors and healthy ageing in Europe: The SENECA Study. Age and Ageing, 32(4), 427-434.

Hogan, C. L., Mata, J., \& Carstensen, L. L. (2013). Exercise holds immediate benefits for affect and cognition in younger and older adults. Psychology and Aging, 28(2), 587-594. doi: 10.1037/a0032634

Johnson, J. M., Sarkisian, N., \& Williamson, J. B. (2015). Using a micro-level model to generate a macro-level model of productive successful aging. Gerontologist, 55(1), 107-119.

Jopp, D., \& Smith, J. (2006). Resources and lifemanagement strategies as determinants of successful aging: On the protective effect of selection, optimization, and compensation. Psychology and Aging, 21 (2), 253-265.

Jopp, D. S., Wozniak, D., Damarin, A. K., De Feo, M., Jung, S., \& Jeswani, S. (2015). How could lay perspectives on successful aging complement scientific theory? Findings from a U.S. and a German life-span sample. Gerontologist, 55(1), 91-106.

Kahana, E., \& Kahana, B. (1996). Conceptual and empirical advances in understanding aging well through proactive adaptation. En
V. L. Bengtson (Ed.), Adulthood and aging. Research on continuities and discontinuities (pp. 18-40). Nueva York: Springer.

Kahana, E., \& Kahana, B. (2001). Successful aging among people with HIV/AIDS. Journal of Clinical Epidemiology, 54, S53. S56.

Kahana, E., Kahana, B., \& Lee, J. E. (2014). Proactive approaches to successful aging: One clear path through the forest. Gerontology, 60(5), 466-474. doi:10.1159/000360222

Kahana, E., Kelley-Moore, J., \& Kahana, B. (2012). Proactive aging: A longitudinal study of stress, resources, agency, and well-being in late life. Aging E) Mental Health, 16(4), 438-451. doi:10.1080/13607863.2011.644519

Kahana, E., Lawrence, R. H., Kahana, B., Kercher, K., Wisniewski, A., Stoller, E., ... Stange, K. (2002). Long-term impact of preventive proactivity on quality of life of the old-old. Psychosomatic Medicine, 64(3), 382-394.

Krueger, K. R., Wilson, R. S., Kamenetsky, J. M., Barnes, L. L., Bienias, J. L., \& Bennett, D. A. (2009). Social engagement and cognitive function in old age. Experimental Aging Research, 35(1), 45-60.

Lang, F. R., \& Carstensen, L. (1994). Close emotional relationships in late life: Further support for proactive aging in the social domain. Psychology and Aging, 9, 315-324.

Lawton, M. P., \& Brody, E. M. (1969). Physical self-maintenance scale (functional assessment). Gerontologist, 9, 179-186.

Llinás, J., Villalta, J. \& López-Pousa, S. (1991). CAMDEX: Adaptación y validación española. Barcelona: Áncora.

Lubben, J. E. (1988). Assessing social networks among elderly populations. Family $\mathbb{E}$ Community Health, 11(3), 42-52.

Martínez, M. R., Hernández, M. J., \& Hernández, M. V. (2006). Psicometría. Madrid: Alianza.

Mendes de León, C. F. (2005). Social engagement and successful aging. European Journal of Aging, 2(1), 64-66. 
Moms, J. C., Heyman, A., Mohs, R. C., Hughes, J. P., Van Belle, G., Fillenbaum, G. D. M. E., ... \& Clark, C. (1989). The Consortium to Establish a Registry for Alzheimer's Disease (CERAD). Part I. Clinical and neuropsychological assesment of Alzheimer's disease. Neurology, 39(9), 1159-1159.

Organización Mundial de la Salud. (2002). Envejecimiento activo: un marco político. Revista Española de Geriatría y Gerontología, 37(S2), 74-105.

Ouwehand, C., \& de Ridder, D., \& Bensing, J. (2007). A review of successful aging models: Proposing proactive coping as an important additional strategy. Clinical Psychology Review, 27(8), 873-884.

Parslow, R. A., Lewis, V. J., \& Nay, R. (2011). Successful aging: Development and testing of a multidimensional model using data from a large sample of older Australians. Journal of the American Geriatrics Society, 59(11), 2077-2083. doi:10.1111/j.1532-5415.2011.03665.x

Potočnik, K., \& Sonnentag, S. (2013). A longitudinal study of well-being in older workers and retirees: The role of engaging in different types of activities. Journal of Occupational Eु Organizational Psychology, 86(4), 497-521. doi:10.1111/joop.12003

Rojo Pérez, F., \& Fernández-Matoralas, G. (Eds.). (2002). Envejecer en casa: la satisfacción residencial de los mayores en Madrid como indicador de su calidad de vida. Madrid: CSIC Consejo Superior de Investigaciones Científicas.

Ryff, C. D. (1989). Beyond Ponce de Leon and life satisfaction: New directions in quest of successful ageing. International Journal of Behavioural Development, 12(1), 35-55.

Rosero-Bixby, L. (2008). The exceptionally high life expectancy of Costa Rican nonagenarians. Demography, 45(3), 673-691.

Rosero-Bixby, L., \& Dow, W. (2005). Costa Rica: Estudio longevidad y envejecimiento saludable (Proyecto N. ${ }^{\circ}$ 828-A4-325). San
José: Universidad de Costa Rica. doi: 10.3886/ICPSR26681

Rosero-Bixby, L., \& Dow, W. H. (2009). Surprising SES Gradients in mortality, health, and biomarkers in a Latin American population of adults. Journals of Gerontology Series B Psychological Sciences and Social Sciences, 64(1), 105-117. doi: 10.1093/ geronb/gbn004

Rowe, J., \& Kahn, R. (1998). Successful aging. Nueva York: Ramdom House.

Sánchez González, D. (2009). Geografía del envejecimiento vulnerable y su contexto ambiental en la ciudad de Granada: discapacidad, dependencia y exclusión social. Cuadernos Geográficos, 45(2), 107-135. Recuperado de https://dialnet.uni rioja.es/descarga/articulo/3123592.pdf

Schaie, K. W. (2005). What can we learn from longitudinal studies of adult development? Research on Human Development, 2(3), 133-158.

Schulz, R., \& Heckhausen, J. (1996). A life span model of successful aging. American Psychologist, 51 (7), 702-714.

Seeman, T., McAvay, G., Merrill, S., Albert, M., \& Rodin, J. (1996). Self-efficacy beliefs and change in cognitive performance: MacArthur studies of successful aging. Psychology and Aging, 11(3), 538-551.

Steverink, N., Lindenberg, S., \& Ormel, J. (1998). Towards understanding successful ageing: Patterned change in resources and goals. Ageing and Society, 18, 441-467.

Strawbridge, W., Cohen, R., Shema, S., \& Kaplan, G. (1996). Successful aging: Predictors and associated activities. American Journal of Epidemiology, 144(2), 135-141.

Tyrovolas, S., Haro, J. M., Mariolis, A., Piscopo, S., Valacchi, G., Tsakountakis, N., \& Metallinos, G. (2014). Successful aging, dietary habits and health status of elderly individuals: A $\mathrm{k}$-dimensional approach within the multi-national MEDIS study. Experimental Gerontology, 60, 57-63. doi: 10.1016/j.exger.2014.09.010 
Vásquez, M., Rodríguez, A., Villarreal, J., \& Campos, A. J. (2014). Relación entre la reserva cognitiva y el enriquecimiento ambiental: una revisión del aporte de las neurociencias a la comprensión del envejecimiento saludable. Cuadernos De Neuropsicología, 8(2), 171-201. doi:10.7714/cnps/8.2.203

Watson, D., Clark, L. A., \& Tellegen, A. (1988). Development and validation of brief measures of positive and negative affect: the PANAS scales. Journal of personality and social psychology, 54 (6), 1063.

Yesavage, J. A., \& Sheikh, J. I. (1986).9/Geriatric depression scale (GDS) recent evidence and development of a shorter version. Clinical gerontologist, 5(1-2), 165-173.

\section{Notas}

* Artículo de investigación. 